\title{
A Generalized Learning Based Framework for Fast Brain Image Registration
}

\author{
Minjeong Kim, Guorong Wu, Pew-Thian Yap, and Dinggang Shen \\ Department of Radiology and BRIC, University of North Carolina at Chapel Hill \\ \{mjkim, grwu, ptyap, dgshen\} @med.unc. edu
}

\begin{abstract}
This paper presents a generalized learning based framework for improving both speed and accuracy of the existing deformable registration method. The key of our framework involves the utilization of a support vector regression (SVR) to learn the correlation between brain image appearances and their corresponding shape deformations to a template, for helping significantly cut down the computation cost and improve the robustness to local minima by using the learned correlation to instantly predict a good subject-specific deformation initialization for any given subject under registration. Our framework consists of three major parts: 1) training of SVR models based on the statistics of image samples and their shape deformations to capture intrinsic image-deformation correlations, 2) deformation prediction for a new subject with the trained SVR models to generate a subject-resemblance intermediate template by warping the original template with the predicted deformations, and 3) estimating of the residual deformation from the intermediate template to the subject for refined registration. Any existing deformable registration methods can be easily employed for training the SVR models and estimating the residual deformation. We have tested in this paper the two widely used deformable registration algorithms, i.e., HAMMER [1] and diffeomorphic demons [2], for demonstration of our proposed frameowrk. Experimental results show that, compared to the registration using the original methods (with no deformation prediction), our framework achieves a significant speedup (6X faster than HAMMER, and $3 \mathrm{X}$ faster than diffeomorphic demons), while maintaining comparable (or even slighly better) registration accuracy.
\end{abstract}

\section{Introduction}

Deformable registration of brain images has been extensively applied for facilitating identification of brain abnormality by comparison between normal and abnormal groups, and for constructing atlases to reflect structural and functional variation of individuals within a population. Most deformable registration methods, regardless intensity- or feature-based, aim to estimate deformation fields for better establishing structural correspondences between images. Although many methods have been previously proposed, determining a reasonable template-subject deformation remains a challenge due to large inter-subject structural variations.

It is worth noting that the traditional registration methods usually lack a good deformation field initialization mechanism. This generally results in long computation 
time arising from the need of estimating large complex deformations, and also vulnerability to misleading matching due to structural ambiguities. To attack these problems, we propose a learning based method to improve registration speed and accuracy by predicting a good initial deformation for bringing the template closer to the given subject. In this way, the remaining deformation from the intermediate template (IT) to the given subject becomes small, and thus can be easily estimated by many deformable registration methods.

Recently, learning-based statistical deformation models [3-5] based on deformation features, i.e., wavelet coefficients, B-spline coefficients, or principal components, have been employed to improve registration accuracy by imposing more realistic registration constraints. Although these approaches have greatly improved registration accuracy, computation cost required for constraining and estimating the deformation field is still quite high.

In this paper, we propose a generalized learning based framework for fast deformable registration, the key of which involves employing support vector regression (SVR) models for rapidly generating intermediate templates. Specifically, we first simulate a large number of training samples with the built image appearance and deformation statistical models. Next, we learn the SVR models for correlating image appearance features and their deformation coefficients (to the template). The learnt SVR models are finally applied to rapidly predict a good initial deformation for a given new subject.

To our knowledge, this current work presents the first attempt to combine, via SVR, the statistics of brain image appearances and their deformation coefficients for effectively guiding deformable registration. It is not difficult to see that many conventional registration algorithms can be easily integrated into our framework for immediate improvement, since our framework is based upon image appearances and deformation coefficients, not on a specific deformation model.

Experimental results on real brain images indicate that this SVR-based deformation prediction approach allows multi-fold speedup of deformable registration algorithms, i.e., $6 \mathrm{X}$ and $3 \mathrm{X}$ faster for HAMMER and diffeomorphic demons, respectively, while retaining similar (or even slightly better) registration accuracy.

\section{Method}

The goal of a deformable registration algorithm is to estimate a dense transformation field $u$ for aligning a subject image $S$ to a template $T$, as shown in Fig. 1. Our approach involves decomposing the overall deformation field $u$ into two parts: the estimated initial deformation field $u_{T \rightarrow I T}$ and the residual deformation field $u_{I T \rightarrow S}$. We determine $u_{T \rightarrow I T}$ automatically with the help of the regression model learnt by statistical models of image appearances and deformation coefficients. To build the statistical models, we first register (using HAMMER, or diffeomorphic demons) a number of training images, to obtain a set of training deformation fields (Fig. 1(a)). PCA is then employed on the deformation fields to capture the principal modes of brain deformations (Fig. 1(b)). A set of brain appearances (Fig. 1(c)), obtained by transforming the training images to the template space, are inverse transformed to the subject 


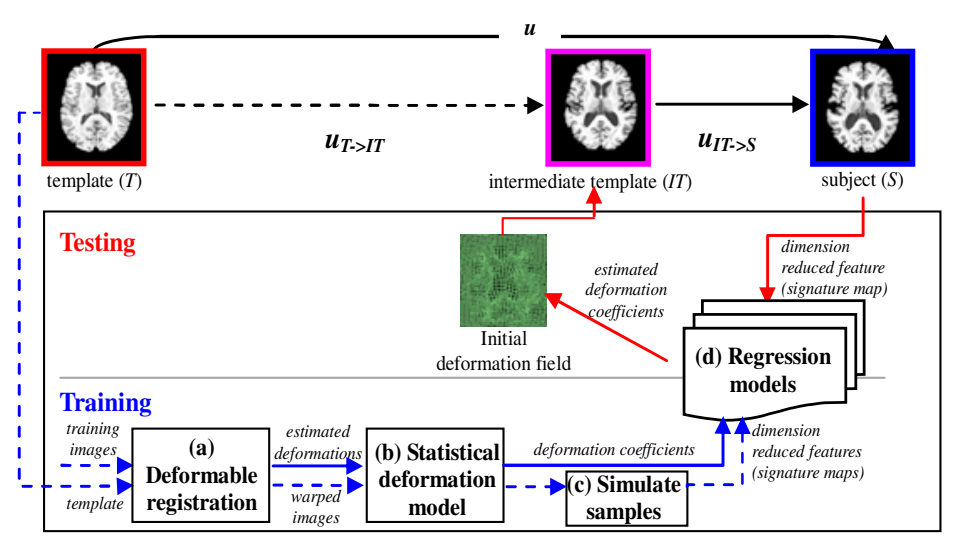

Fig. 1. Schematic illustration of the proposed registration framework

space by the deformation fields generated via perturbing the PCA coefficients (for generating a large number of samples for training). Using these samples, SVR models, bridging the intrinsic appearance-deformation statistics, are trained (Fig. 1(d)). Then, given a new subject, an initial deformation field, as well as the corresponding intermediate template, can be instantly predicted by the trained SVR models (Fig. 1(d)). Finally, the estimation of residual deformations from the intermediate template to the new subject can be performed at a significantly reduced computation cost due to their similarity. It is worth noting that all existing registration methods can be used for the preparation of training deformation fields (Fig. 1(a)), and for refining the registration from the intermediate template to the new subject $u_{I T \rightarrow S}$. Details on statistical deformation and appearance models, sample simulation, and construction of SVR models will be given in the following subsections.

\subsection{Statistical Deformation-Appearance Model for Sample Simulation}

A sufficiently large set of training samples, covering an adequate space of brain shape variation, is important for effective training of the SVR models. Instead of simulating deformation fields based only on some statistical deformation models, as was previously done [6], we design a statistical model which incorporates both deformation and appearance information for simulating training samples.

Statistical deformation model: Given a set of $M$ brain images $I_{0}$, their respective deformation fields $U_{0}$ can be estimated by a deformable registration algorithm such as HAMMER or diffeomorphic demons. By applying PCA on $U_{0}$, we can obtain a statistical deformation model to capture brain shape variations. Specifically, the eigenvectors of deformation covariance matrix represent the principal modes of variation, and their eigenvalues indicate the magnitude of deformation variation along the direction of each corresponding eigenvector. To characterize the principal shape changes, the eigenvectors with the top $Q$ largest eigenvalues are used for approximate representation of the original deformations. The reconstructed counterpart $\hat{u}_{i, 0}$ of $u_{i, 0} \in U_{0}$ can be written as: 


$$
\hat{u}_{i, 0} \approx \bar{u}+\sum_{q=1}^{Q} c_{i, 0}^{q} \sqrt{\lambda_{u}^{q}} \Phi_{u}^{q},
$$

where $\bar{u}$ denotes the mean deformation field, and $\lambda_{u}^{q}$ and $\Phi_{u}^{q}$ are the eigenvalues and eigenvectors of the deformation covariance matrix. Each deformation field $u_{i, 0}$, is represented in a subspace spanned by $Q$ the eigenvectors using a deformation coefficient (column) vector $\vec{c}_{i, 0}=\left[\begin{array}{lllll}c_{i, 0}^{1} & \ldots & c_{i, 0}^{q} \ldots & c_{i, 0}^{Q}\end{array}\right]^{T}$, where $c_{i, 0}^{q}=\left(\Phi_{u}^{q}\right)^{T}(u-\bar{u}) / \sqrt{\lambda_{u}^{q}}$.

Given that we are often faced with the problem of having a limited number of training samples in $I_{0}$, we simulate additional brain images based on the statistical model of $U_{0}$, to increase the robustness of estimating the brain appearance model. We generate $N$ new deformation coefficient vectors $\vec{c}_{i, j}(i=1 \ldots M, j=1 \ldots N)$ for each coefficient vector $\vec{c}_{i, 0}$ by perturbing it with $\vec{p}_{i, j}$, i.e., $\vec{c}_{i, j}=\vec{c}_{i, 0}+\vec{p}_{i, j}$. The range of each element of the perturbation vector $\vec{p}_{i, j}$ is determined from the mean and standard deviation of the training deformation fields. Thus, a total of $M(N+1)$ simulated deformation fields $\tilde{u}_{i, j}$ can be generated as below:

$$
\tilde{u}_{i, j}=\bar{u}+\sum_{q=1}^{Q}\left(c_{i, 0}^{q}+p_{i, j}^{q}\right) \sqrt{\lambda_{u}^{q}} \Phi_{u}^{q}
$$

Statistical brain appearance model: To build a more efficient statistical model which is capable of estimating deformation coefficients based on image features, we further enlarge the sample dataset by incorporating various brain appearances. Specifically, we first align a set of $M$ training images $I_{0}$ onto the template space to get a set of $M$ warped images in the template space. We then generate a series of new brain images $\tilde{I}_{i, k}(i=1 \ldots M, k=1 \ldots M(N+1))$ by inverse deforming those $M$ warped images from the template space to the individual spaces by the $M(N+1)$ deformation fields generated by Eq. 2. By including $M$ initial training samples, our statistical model can generate a total of $M^{2}(N+1)$ samples, thus able to characterize a wide spectrum of deformation and image appearance.

In order to obtain an appearance model feasible for training the regression models, we employ a few strategies to reduce the dimensionality of $\tilde{I}$ before building the appearance model. For each sample image $\tilde{I}_{i, k} \in \tilde{I}$, the background voxels will be first cropped away and then the remained image will be down-sampled, obtaining $\tilde{I}_{i, k}^{\prime}$. To better represent the shape variation of each sample image $\tilde{I}_{i, k}^{\prime}$, we further extract brain boundaries along the interfaces between WM, GM, and CSF as the shape descriptors to construct a signature image $S I_{i, k}$. Next, we apply PCA to each signature image $S I_{i, k}$ to represent it by a (low) $D$-dimensional column vector $\overrightarrow{S m}_{i, k}$, called as signature map $(S M)$.

\subsection{Construction of SVR Models}

After obtaining the deformation coefficient vectors $\vec{c}_{i, k}$ and their corresponding signature maps $\overrightarrow{s m}_{i, k}$, we start to learn their correlations by support vector regression (SVR) models. Specifically, we train $Q$ SVR models, with each responsible for learning the non-linear regression of each row of the deformation coefficient matrix 
$C_{Q \times(M+M N)}=\left[\begin{array}{lll}\ldots & \left.\vec{c}_{i, k} \ldots\right] \\ & \text { with respect to the signature maps }\end{array}\right.$ $S M_{D \times(M+M N)}=\left[\ldots \overrightarrow{S m}_{i, k} \ldots\right]$. SVR is a supervised learning technique for finding nonlinear mapping functions which correlate a number of input variables (features) to a continuous output variable (targets). The features in our case are therefore the signature maps $S M_{D \times(M+M N)}$ and the targets are the rows of the deformation coefficient matrix $C_{Q \times(M+M N)}$. We note here that a common set of signature maps $S M$ is used as the features for all regression models, while the $q^{\text {th }}$ row of deformation coefficient matrix $C$ is used independently as the targets for the $q^{\text {th }}$ regression model.

For the nonlinear regression, we use a radial basis function (RBF) as the kernel function, and optimize several SVR meta-parameters for building more reliable regression models. We first estimate the kernel size based on the distribution of each signature map $\overrightarrow{s m}_{i, k}$, i.e., the average of the distances from all possible pairs of $\overrightarrow{s m}_{i, k}$. To achieve the global minimum with reliable generalization bound of the regression function, we optimize the parameters $\varepsilon$ and $\gamma$. Parameter $\varepsilon$ is used to control the width of the insensitive zone which penalizes the training data outside this zone. Constant $\gamma(>0)$ determines the trade-off between the flatness of the regression function and the tolerance to deviation larger than $\varepsilon$. The value of $\varepsilon$ and $\gamma$ can be calculated as [7]:

$$
\begin{gathered}
\varepsilon=\tau \cdot \sigma_{s} \sqrt{\ln (M+M N) /(M+M N)} \\
\gamma=\max \left(\left|\bar{c}+3 \sigma_{c}\right|,\left|\bar{c}-3 \sigma_{c}\right|\right),
\end{gathered}
$$

where $\sigma_{s}$ is the standard deviation of distances between all pairs of $\overrightarrow{s m}_{i, k}, \tau$ is an empirical constant, and $M+M N$ is the number of signature maps used as features for regression. $\bar{c}$ and $\sigma_{c}$ are the mean and the standard deviation of the deformation coefficients $\vec{c}_{i, k}$, respectively.

\subsection{Deformation Prediction for New Subject}

After training the regression models, a good initial deformation can be rapidly predicted for any given subject, to bring the template close to the given subject and thus facilitate fast and robust registration. Specifically, for a given subject $S$, its signiture map $\overrightarrow{s m}_{S}$ will be first computed by projection onto the $Q$ top eigenvectors (Section 2.2) after affine alignment to the template by FSL FLIRT. The deformation coefficients $\vec{c}_{s}=\left(c_{s}^{1} c_{s}^{2} \ldots c_{s}^{Q}\right)$ can then be predicted one by one through each SVR model. Next, it is straightforward to obtain the initial deformation $u_{T \rightarrow I T}$ for subject $S$ by Eq. 1 and also the corresponding intermediate template by warping the template with respect to $u_{T \rightarrow I T}$. Now we only need to estimate the residual deformation from the intermediate template to the subject, instead of the original template to the subject as done in many conventional registration algorithms. This step helps save a significant amount of computation cost for overall registration, and also circumvents the errorprone approach of estimating large deformations from the original template to the subject. After estimating $u_{I T \rightarrow S}$ (by a conventional registration method, i.e., HAMMER or diffeomorphic demons), $u_{T \rightarrow I T}$ and $u_{I T \rightarrow S}$ are concatenated to form a final deformation field, from the template to the subject. 


\section{Experimental Results}

We use both HAMMER and diffeomorphic demons for evaluation of our framework and demonstration of its generality. Note that our intention here is not to compare HAMMER and diffeomorphic demons.

\subsection{Deformation Prediction}

We select randomly $M=50$ images from a MR brain image database for training. By using both HAMMER and diffeomorphic demons, we first estimate the deformation fields of all these images with respect to a selected template (Fig. 2a). By applying PCA, we can build a statistical deformation model with 49 modes from the 49 eigenvectors with non-zero eigenvalues. For denser sampling of the deformation field space, we apply $N=4$ perturbations, thus giving us a total of $M(N+1)=250$ deformation samples, and $M^{2}(N+1)=12500$ brain image samples by inverse deforming those 50 aligned images from the template space to the individual spaces. We then use half (i.e., 6250) of the deformation-image pairs for training the SVR models, and the other half for testing. The averaged prediction error for all deformation coefficients on the training and testing data is $0.1 \%$ and $7.8 \%$, respectively. Since the testing data is not used for training, its error is larger than the training error, which is reasonable. This result also indicates that our model is able to predict very good initial deformations for the testing data, which can significantly help reduce the computation cost and registration robustness as reported below.

Given a new subject, we can align it linearly to the template space, and further build its signature map by down-sampling, feature extraction and dimensionality reduction. Then, we can use the SVR models, constructed in the training stage, to predict the initial deformation for the subject. To evaluate the quality of the predicted deformations, we use 50 new images (not used for training) to show their predicted intermediate templates in Fig. 2. These results indicate that our method can predict very good

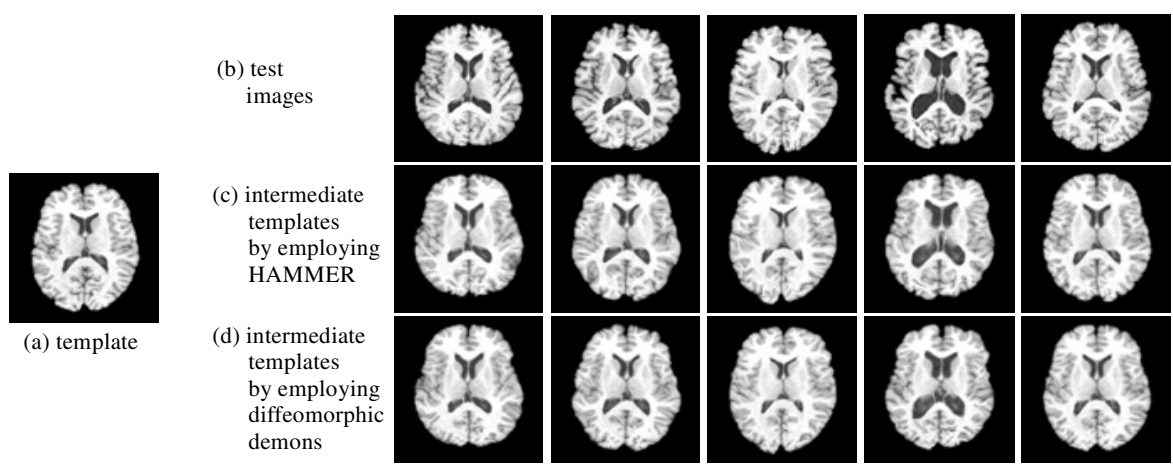

Fig. 2. Demonstration of the intermediate templates (c) and (d) estimated for 5 test images (b), by using HAMMER and diffeomorphic demons, respectively. Compared to the original template (a), the intermediate templates by both methods are very close to the test images, implying that our framework has generality for incorporation of different registration methods. 
intermediate templates for both HAMMER and diffeomorphic demons algorithms, conforming its generality for incorporation of different registration methods.

\subsection{Registration Performance}

Twenty images are separately registered using HAMMER and diffeomorphic demons with and without deformation initialization by our method. The registered images are then averaged to give a visual indication of the registration accuracy, as shown in Fig. 3. As can be observed, the average brain images yielded by the algorithms with and without our framework (i.e., (b) and (c) for HAMMER, and (d) and (e) for diffeomorphic demons) are comparable. We note especially that the average brain given by HAMMER using our framework is slightly sharper than that of the original HAMMER, especially in the cortical regions.

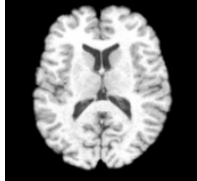

(a)

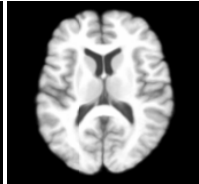

(b)

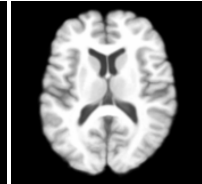

(c)

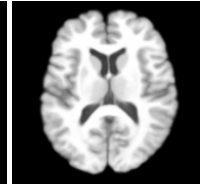

(d)

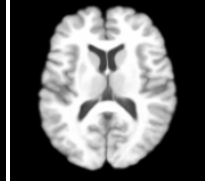

(e)

Fig. 3. Average brain images constructed from 20 brain images aligned by HAMMER and diffeomorphic demons with and without our framework. (a) template; (b) by HAMMER; (c) HAMMER with our framework; (d) diffeomorphic demons; and (e) diffeomorphic demons with our framework.

The registration accuracy is further evaluated using images from NIREP database. Since gray matter regions of brain MRIs in this database were manually segmented into 32 ROIs, we use them to compute the average ROI overlap ratio after registration for accuracy evaluation. However, this database contains only $16 \mathrm{MRI}$ brain images, and we therefore include more images from the OASIS database for training. We select one image from NIREP as the template and register 50 images from OASIS to the selected template, using HAMMER and diffeomorphic demons, respectively, for training our model. Then, we use the remaining 15 images from NIREP for testing the registration performance, by computing the average overlap ratio of the ROIs between the template and each of the 15 aligned images. The average overlap ratio of each ROI is shown in Fig. 4. The overall overlap ratio yielded by HAMMER alone is $66 \%$, and with use of our framework its performance is slightly increased to $67 \%$. The overall overlap ratio given by diffeomorphic demons is $64 \%$, and with used of our framework its performance is increased to $66 \%$. These results show that the use of our framework can slightly improve the registration accuracy, with significant speed improvement as mentioned below. We also note that, although the training and testing sets come from different sources, our proposed method is still capable of yielding good performance. 

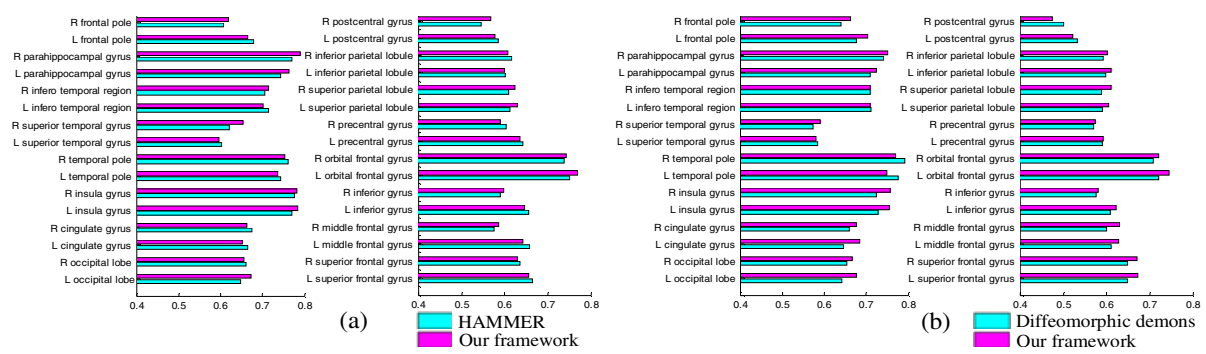

Fig. 4. Average overlap ratios of aligned ROIs. (a) HAMMER with and without our framework, and (b) diffeomorphic demons with and without our framework.

\subsection{Speed}

Lower computation cost requirement is one of the key advantages of our proposed framework. We use 50 test images of size $256 \times 256 \times 198$ to compare the average deformation estimation time of HAMMER and diffeomorphic demons, with and without use of our framework. For similar registration accuracy, HAMMER alone took 90 minutes, and the computation time is reduced to 16 minutes with use of our framework. This indicates about six fold of speed improvement. Diffeomorphic demons alone took 219 seconds, and our framework can help reduce the overall computation time by threefold to 71 seconds.

\section{Conclusion}

A fast deformable brain registration framework using a novel deformation prediction model has been presented. Specifically, regression models are trained to capture the correlations between image appearances and deformation coefficients. The learnt correlation models are then used to predict rapidly a good initial deformation, as well as the corresponding intermediate template, for any given image. Since the shape difference between the intermediate template and the given image becomes small, the conventional registration methods (i.e., HAMMER and diffeomorphic demons) when incorporated into our framework can perform much faster with comparable accuracy. Future work includes testing more registration methods in the proposed framework such as the B-spline based registration methods.

\section{References}

[1] Shen, D., Davatzikos, C.: HAMMER: Hierarchical attribute matching mechanism for elastic registration. IEEE Transactions on Medical Imaging 21, 1421-1439 (2002)

[2] Vercauteren, T., Pennec, X., Perchant, A., Ayache, N.: Diffeomorphic demons: Efficient non-parametric image registration. NeuroImage 45, S61-S72 (2009)

[3] Xue, Z., Shen, D., Davatzikos, C.: Statistical Representation of High-Dimensional Deformation Fields with Application to Statistically-Constrained 3D Warping. Medical Image Analysis 10, 740-751 (2006) 
[4] Rueckert, D., Frangi, A., Schnabel, J.: Automatic construction of 3-D statistical deformation models of the brain using nonrigid registration. IEEE Transactions on Medical Imaging 22, 1014-1025 (2003)

[5] Loeckx, D., Makes, F., Vandermeulen, D., Suetens, P.: Non-rigid image registration using a statistical spline deformation model. In: Taylor, C.J., Noble, J.A. (eds.) IPMI 2003. LNCS, vol. 2732, pp. 463-474. Springer, Heidelberg (2003)

[6] Tang, S., Fan, Y., Wu, G., Kim, M., Shen, D.: RABBIT: rapid alignment of brains by building intermediate templates. Neuroimage 47, 1277-1287 (2009)

[7] Cherkassky, V., Ma, Y.: Selection of meta-parameters for support vector regression. In: Dorronsoro, J.R. (ed.) ICANN 2002. LNCS, vol. 2415, pp. 687-693. Springer, Heidelberg (2002) 